\title{
The Baseline Characteristics of Patients with COVID-19 and Impact of Various Risk Factors on the Outcome of Patients Admitted in Tertiary Care Hospital.
}

Samiullah Shaikh ( $\nabla$ samiullahshkh7@gmail.com )

Liaquat University of Medical and Health Sciences

Faiza Deedar

Liaquat University of Medical and Health Sciences

Tara Chand Devrajani

Liaquat University of Medical and Health Sciences

\section{Research}

Keywords: Covid-19, Gram-Covid score, Diabetes mellitus, Hypertension, Obesity

Posted Date: March 18th, 2021

DOl: https://doi.org/10.21203/rs.3.rs-305334/v1

License: (c) (i) This work is licensed under a Creative Commons Attribution 4.0 International License.

Read Full License 


\section{Abstract}

Background: The outbreak caused by novel coronavirus was first reported in Wuhan, China in December 2019 when a new pathogen was discovered from the bronchoalveolar lavage fluid of the patient suffering from pneumonia. Globally over 81.47 million confirmed cases and 1.79 million deaths has been reported. The clinical spectrum ranges from mild influenza-like illness to severe life threatening respiratory disease requiring ventilatory support to death. The purpose of this study was to evaluate the baseline carecteristic of the patients and impact of various risk factors on the outcome of patients admitted in tertiary care hospital.

\section{MATERIALS AND METHODS}

Study Area and Period: This study was conducted at Liaquat University Hospital from April 2020 to September 2020.

Study Design: Cross-Sectional Descriptive.

Inclusion Criteria: The patients were included on the basis of diagnosis of COVID-19 confirmed on reverse transcription Polymerase Chain reaction (RT-PCR) test.

Categorical variables such as age, sex, Gram-Covid risk category ,diabetes mellitus, hypertension, renal, pulmonary disease, obesity, outcome of patient were presented as frequency and percentage

RESULTS: This study included 235 consecutive patients admitted at Liaquat University Hospital from April 2020 to September 2020. There were 187(79.6\%) male and 48(20.4\%) female. The age < 65 years were present in 80 (34\%) and $\geq 65155(66 \%)$ patients. The most prevalent symptoms were fever ,breathlessness, cough, anorexia, fatigue in all $235(100 \%)$ patients. Severity of illness according to GramCovid Score 18 (7.7\%) patients were in low-risk group, 93(39.6\%) in moderate risk group and 124 (52.8\%) in High risk group.

There were no risk factors in $121(51.5 \%)$, one risk factor in $49(20.9 \%)$, two risk factors in $32(13.6 \%)$, three risk factors in 18 ( $7.7 \%)$ and four risk factors in $15(6.4 \%)$ patients.

The outcome of patients showed $57(24.3 \%)$ deaths, 107 (45.5\%) patients had prolong stay and $71(30.2 \%)$ patients recovered. A strong relationship was observed between the outcome of patient with age, number of risk factors, severity of illness according to gram - Covid score whereas insignificant relationship with the sex of patients.

Conclusion: COVID-19 patients clinically present with fever, cough and breathlessness. Majority of patients present with moderate to high severity. Patient with advanced age, high risk on gram-Covid score with multiple comorbidities are particularly susceptible to adverse outcome.

\section{Introduction}


The outbreak caused by novel coronavirus was first reported in Wuhan, China in December 2019 when a new pathogen was discovered from the bronchoalveolar lavage fluid of the patient suffering from pneumonia. ${ }^{1}$ The said pathogen had the similar features of the coronavirus family and therefore was classified in the subgenus Sarbecovirus, Orthocoronavirinae subfamily. The virus is named as severe acute respiratory distress syndrome coronavirus 2 and the disease caused by it is named as coronavirus disease 2019" (COVID-19). ${ }^{2}$

World Health Organization on 11th March 2020 decalared Coronavirus Disease(COVID-19) caused by Coronavirus-2 as pandemic and upto 30th September 2020 at the closure of study globally over 32.7 million confirmed cases and 991000 deaths has been reported. ${ }^{3,4}$ The incubation period of Coronavirus disease ranges from 2 to 14 days with clinical spectrum varying from mild influenza-like illness to severe life threatening respiratory disease requiring ventilatory support to death. It has been reported recently that nearly 14 to $29 \%$ hospitalized patients with COVID-19 pneumonia require intensive care support for respiratory support for hypoxic respiratory failure, with acute respiratory distress syndrome (ARDS) developing in $33 \%$ of hospitalized patients within 8 days of onset of symptoms. 5,6

The most common symptoms of COVID-19 are cough, fever, and breathlessness. Gastrointestinal symptoms such as diarrhea, nausea or vomiting are also common. ${ }^{7}$

It has now been observed that old age and other comorbidities are potential risk factors for the worse outcome of COVD-19 patients. ${ }^{8}$ According to preliminary data nearly $30 \%$ patients were $>65 \%$ years of age and nearly $25 \%$ patients had anyone underling medical condition. Among the common medical conditions include diabetes mellitus, hypertension, cardiovascular disease, obesity and chronic pulmonary ,renal disease and the severity of disease at admission. But the impact of each factor has not been asessed leading to variability in management and outcome. ${ }^{5,9}$

The purpose of this study was to evaluate the baseline carecteristic of the patients suffering from COVID19 and impact of various risk factors on the outcome of patients admitted in a tertiary care hospital.

\section{Materials And Methods}

2.1. Subjects: This cross-sectional study included 235 consecutive adults ( $>18$ years age) of either sex admitted at Liaquat University Hospital from April 2020 to September 2020. The patients were included on the basis of diagnosis of COVID-19 confirmed on reverse transcription Polymerase Chain reaction (RTPCR) test. The approval for study was obtained from University ethical committee.

All patients were admitted in High Dependency Unit (HDU) and shifted to Intensive care Unit (ICU) specified for COVID-19 patients based on the following criteria: ${ }^{10}$

1. Respiratory rate $>40$ breaths $/ \mathrm{min}$. 
2. Pulse oxygen saturation (PSO2) $<90 \%$ or $\mathrm{PO} 2<60 \mathrm{mmHg}$ while the patient was breathing oxygen at a flow rate equal or $>7 \mathrm{~L} / \mathrm{min}$ for $>30$ minutes.

3. Hemodynamic instability and use of vasopressors.

4. Glasgow Coma Scale of 12 points or lower.

5. Requiring continuous renal replacement therapy.

\subsection{Data Collection:}

The COVID team supervised by Physician was responsible for collecting the following data

1. Demographic data such as gender, age, occupation, date of onset of symptoms.

2. Comorbidities : such as Diabetes Mellitus, Hypertension, Ischemic heart Disease, Pulmonary, Renal diseases and Obesity.

3. Results of laboratory tests:

4. Outcomes such as : Recovery, Prolong stay at hospital and death.

2.3. Demographic: Data: A semisynthetic form was designed for collection of data on age, sex, comorbidity (hypertension, diabetes, cardiovascular disease, chronic kidney disease), symptoms from onset to hospital admission (fever, cough, expectoration, dyspnea, muscle pain, headache, sore throat, chill, diarrhea, fatigue)

2.4. Laboratory investigations: Blood $\mathrm{CBC}$, Blood sugar,HbA1c,urea and serum creatinine, serum electrolytes, liver function test, lactate dehydrogenase, creatine kinase, D-Dimer, procalcitonin, brain natriuretic peptide, lactate dehydrogenase (LDH) , arterial blood gases (ABG) , SARS-COVID 19 PCR were performed at Liaquat University Research Lab.

X-ray chest, ECG, CT-Chest (optional) , echocardiography(optional) , Ultrasound abdomen (optional) were performed by the radiology department of Liaquat University hospital,

\subsection{Treatment}

The patients were given

1. Supplemental oxygen, High flow nasal cannula(HFNC), Non-invasive ventilation (NIV), mechanical ventilation depending on the severity of hypoxia.

\section{Anti-biotics}

3. Steroids

4. Anticoagulants.

5. Anti viral therapy. 


\subsection{Definitions:}

1.Acute respiratory distress syndrome (ARDS) : was defined by the Berlin definition which requires that all of the following criteria be present for diagnosis: ${ }^{11}$

- Respiratory symptoms must have begun within one week of a known clinical insult, or the patient must have new or worsening symptoms during the past week.

- Bilateral opacities must be present on a chest radiograph or computed tomographic (CT) scan not explained by pleural effusions, lobar collapse, lung collapse, or pulmonary nodules.

- Oxygenation A moderate to severe impairment of oxygenation must be present, as defined by the ratio of arterial oxygen tension to fraction of inspired oxygen $\left(\mathrm{PaO}_{2} / \mathrm{FiO}_{2}\right)$. The severity of the hypoxemia defines the severity of the ARDS:

- Mild ARDS - The $\mathrm{PaO}_{2} / \mathrm{FiO}_{2}$ is $>200 \mathrm{mmHg}$, but $\leq 300 \mathrm{mmHg}$, on ventilator settings that include positive end-expiratory pressure (PEEP) or continuous positive airway pressure (CPAP) $\geq 5 \mathrm{~cm} \mathrm{H}_{2} \mathrm{O}$.

- Moderate ARDS - The $\mathrm{PaO}_{2} / \mathrm{FiO}_{2}$ is $>100 \mathrm{mmHg}$, but $\leq 200 \mathrm{mmHg}$, on ventilator settings that include PEEP $\geq 5 \mathrm{~cm} \mathrm{H}_{2} \mathrm{O}$.

- Severe ARDS - The $\mathrm{PaO}_{2} / \mathrm{FiO}_{2}$ is $\leq 100 \mathrm{mmHg}$ on ventilator settings that include PEEP $\geq 5 \mathrm{~cm} \mathrm{H}_{2} \mathrm{O}$.

2. Diabetes Mellitus: The diagnosis of Diabetes mellitus was based on Fasting Plasma Glucose of 126 $\mathrm{mg} / \mathrm{dl}(7.00 \mathrm{mmol} / \mathrm{L})$ or higher, $\mathrm{Hb} \mathrm{A}_{1 \mathrm{C}}$ of $6.5 \%$ or higher and 2-hour value of Oral Glucose Tolerance test of $200 \mathrm{mg} / \mathrm{dl}(11.1 \mathrm{mmol} / \mathrm{L})$ or higher is defined as diabetes, ${ }^{12}$

3. Hypertension: The diagnosis of hypertension was based on the "2020 International Society of Hypertension Global Hypertension Practice Guidelines. ${ }^{13}$

\section{Acute kidney injury (AKI) Definition:}

Acute kidney injury was defined according to the KDIGO clinical practice guidelines; as one of the following: ${ }^{14}$

- An increase in serum creatinine by $\geq 0.3 \mathrm{mg} / \mathrm{dl}$ ( $\geq 26.5 \mu \mathrm{mol} / \mathrm{l})$ within $48 \mathrm{~h}$

- An increase in serum creatinine to $\geq 1.5$ times baseline within the previous 7 days

- Urine volume $\leq 0.5 \mathrm{ml} / \mathrm{kg} / \mathrm{h}$ for $6 \mathrm{~h}$.

5. Chronic obstructive pulmonary disease (COPD): is the name for a group of lung conditions that cause breathing difficulties. ${ }^{15}$

It includes:

- emphysema - damage to the air sacs in the lungs 
- chronic bronchitis - long-term inflammation of the airways

6. Body Mass Index (BMI): is a person's weight in kilograms divided by the square of height in meters. A high BMI can be an indicator of high body fatness.

Obesity is frequently subdivided into categories: ${ }^{16}$

- Class 1: BMI of 30 to $<35$

- Class 2: BMI of 35 to $<40$

- Class 3: BMl of 40 or higher. Class 3 obesity is sometimes categorized as "extreme" or "severe" obesity.

\section{Severity of Illness:}

The severity of illness was assessed by COVID-GRAM Critical Illness Risk Score which included the following variables. ${ }^{17}$

1. X-ray abnormality

2. Age

3. Hemoptysis

4. Dyspnea

5. Unconsciousness

6. Number of comorbidities

Includes COPD, hypertension, diabetes, coronary heart disease, chronic heart disease, chronic kidney disease, cancer, cerebral vascular disease, Hepatitis B, and immunodeficiency

7. Cancer history

8. Lactate dehydrogenase

9. neutrophil-lymphocyte ratio

10. Direct bilirubin

The results of above variables were placed in calculator available on the website https://www.mdcalc.com/covid-gram-critical-illness-risk-score.

Based on the results of patients were categorized into low, moderate and high risk group.

8. Outcomes 18,19 
Recovery: According to WHO recovery time described as return to baseline health after hospitalization is two weeks.

Prolong stay: who have ongoing shortness of breath, some requiring supplemental oxygen, or with persistent chest pain on exertion, blood clotting problems, poor concentration, gastrointestinal distress, and reduced muscle strength and impaired grasping power after two weeks of hospital admission.

\section{COVID-19-Related Deaths :}

The case-fertility is defined as the proportion of COVID-19 related deaths among patients who were COVID-19 positive on PCR irrespective of pre-existing diseases that may have caused death.

\section{Statistical Analysis}

Categorical variables such as age, sex, Gram-Covid risk category,diabetes mellitus, hypertension, renal, pulmonary disease, obesity, outcome of patient were presented as frequency and percentage .

The predictive accuracy of age,sex,severity of illness and comorbidities related to outcome was tested by measuring the area under curves receiver oprating characteristic (AUCROC). Based on ROC, the best cutoff points was chosen. Diagnostic accuracy was evaluated by calculating the sensitivity, specificity, positive predictive value and negative predictive value (PPV,NPV). ROC curve comparison was performed which uses calculation of the area under curve and $95 \%$ confidence interval (Cls) by the technique described by Hanley and McNeil. ${ }^{20}$ The main end point was to observe the relationship of age, gender, severity of illness and comorbidities with the outcome of patients. We used SPSS (version 22.0) for all analyses. A $p<0.05$ was considered as statistically significant.

\section{Results}

\subsection{Demographics, Symptoms, co-morbidities, Severity of illness and outcome of COVID-19 patients:}

This study included 235 consecutive patients admitted in HDU/ ICU from April 2020 to September 2020. There were $187(79.6 \%)$ male and $48(20.4 \%)$ female. The age $<65$ years were present in $80(34 \%)$ and $\geq$ $65155(66 \%)$ patients. The most prevalent symptoms were fever ,breathlessness, cough, anorexia, fatigue in all $235(100 \%)$ patients.Severity of illness according to Gram-Covid Score showed 18 (7.7\%) patients were in low-risk group, 93(39.6\%) in moderate risk group and 124 (52.8\%) in high risk group. There were no co-morbidity in $121(51.5 \%)$, one co-morbidity in 49(20.9\%), two co-morbidities in 32 (13.6\%), three comorbidities in 18(7.7\%) and four co-morbidities in $15(6.4 \%)$ patients.Diabetes mellitus was present in 88/235 (37.4\%), Hypertension in 80/235(34 \%) patients, Cardiovascular disease (CVD) in 33/235(14\%), Obesity in 20 (8.9\%), Chronic Obstructive Airway Disease (COPD) in 30/235 (12.8\%) and Renal disease in $14 / 235$ (6\%) patients. The outcome showed 57/235(24.3\%) deaths, 107/235 (45.5\%) had prolong stay in hospital and 71/235(30.2\%) recovered and discharged from hospital within two weeks time. 
Table No:1 Demographics, Symptoms, comorbidities, Severity of illness and outcome of COVID-19 patients(235). 


\begin{tabular}{|c|c|c|}
\hline Variable & Frequency & Percentage(\%) \\
\hline \multicolumn{3}{|l|}{ Sex } \\
\hline Male & 187 & 79.6 \\
\hline Female & 48 & 20.4 \\
\hline \multicolumn{3}{|l|}{ Age: } \\
\hline$<65$ years & 80 & 34 \\
\hline$>65$ & 155 & 66 \\
\hline \multicolumn{3}{|l|}{ Symptoms } \\
\hline fever , & 235 & 100 \\
\hline breathlessness, & 235 & 100 \\
\hline cough, & 235 & 100 \\
\hline anorexia, & 235 & 100 \\
\hline fatigue & 235 & 100 \\
\hline \multicolumn{3}{|l|}{ Co-Morbidities: } \\
\hline No & 121 & 51.5 \\
\hline One & 49 & 20.9 \\
\hline Two & 32 & 13.6 \\
\hline Three & 18 & 7.7 \\
\hline Four & 15 & 6.4 \\
\hline \multicolumn{3}{|l|}{ Individual co-Morbidities: } \\
\hline Diabetes mellitus & 88 & 37.4 \\
\hline Hypertension & 80 & 34 \\
\hline Cardiovascular disease (CVD) & 33 & 14 \\
\hline Chronic Obstructive Airway Disease (COPD) & 30 & 12.8 \\
\hline Obesity & 21 & 8.9 \\
\hline Renal Disease & 14 & 06 \\
\hline
\end{tabular}




\begin{tabular}{|lll|}
\hline Variable & Frequency & Percentage(\%) \\
\hline Severity of Illness & & \\
Gram-Covid Score & 18 & 7.7 \\
Low-risk group, & 93 & 39.6 \\
Moderate risk group & 124 & 52.8 \\
High risk group. & & \\
\hline Outcome: & & \\
Deaths & 57 & 24.3 \\
prolong stay & 107 & 45.5 \\
Recovery & 71 & 30.2 \\
\hline
\end{tabular}

\subsection{COVID-19 patients' Laboratory Findings:}

Creatinine Phosphokinase $(\mathrm{CPK})<2 \times$ the upper limit of normal (normal range: 40 to 150 units/L) in $128(54.5 \%)$ and $>2 x$ the upper limit of normal was present in $107(45.4 \%)$ patients. CRP $<100 \mathrm{mg} / \mathrm{L}$ was present in 154 ( $65.5 \%$ ) and $>100 \mathrm{mg} / \mathrm{L} 81$ (34.5\%) patients. Ferritin level < $500 \mathrm{mcg} / \mathrm{L}$ (normal range: females 10 to $200 \mathrm{mcg} / \mathrm{L}$; males 30 to $300 \mathrm{mcg} / \mathrm{L}$ ) was present $154(65.5 \%)$ and $>500 \mathrm{mcg} / \mathrm{L}$ in $81(34.5 \%)$ patients. D-Dimer $<1000 \mathrm{ng} / \mathrm{mL}$ was present in $184(82.6 \%)$ and $>1000 \mathrm{ng} / \mathrm{mL}$ in $41(17.4 \%)$ patients. Troponin T $<2 \times$ the upper limit of normal (normal range females 0 to $9 \mathrm{ng} / \mathrm{L}$; males 0 to 14 $\mathrm{ng} / \mathrm{L})$ in $199(84.7 \%)$ and $>2 \times$ the upper limit of normal in 36(15.3\%) patients. Lactic Dehydrogenase $(\mathrm{LDH})<245$ units/L (normal range: 110 to 210 units/L) in 148(63\%) and $>245$ units/L in 87( $37 \%$ ) patients. Procalcitonin Normal range $(0.15-2 \mathrm{ng} / \mathrm{mL})$ was $<2$ in $181(77 \%)$ and $>2$ in 54 (23\%) patients. The neutrophil / lymphocyte ratio 1-3 in 72 (30.6\%),3-9 in 89 (37.8) and >9 in 74(31.4) patients. Table No: 2 shows the laboratory workup of patients.

Table No:2 Laboratory workup of Patients (235). 
Creatinine Phosphokinase

( $\mathrm{CPK}$ )

(Normal range: 40 to 150 units/L)

$1 .<2 \times$ the upper limit of normal

128

54.5

2. $>2 x$ the upper limit of normal

107

45.4

CRP

$1 .<100 \mathrm{mg} / \mathrm{L}$

154

65.5

2. $>100 \mathrm{mg} / \mathrm{L}$

81

34.5

Ferritin level

(normal range: females 10 to $200 \mathrm{mcg} / \mathrm{L}$; males 30 to $300 \mathrm{mcg} / \mathrm{L}$ )

$1 .<500 \mathrm{mcg} / \mathrm{L}$

154

65.5

2. $>500 \mathrm{mcg} / \mathrm{L}$ 81

D-Dimer

1. $<1000 \mathrm{ng} / \mathrm{mL}$

184

82.6

2. $>1000 \mathrm{ng} / \mathrm{mL}$

41

17.4

Troponin T

(normal range females 0 to $9 \mathrm{ng} / \mathrm{L}$; males 0 to $14 \mathrm{ng} / \mathrm{L}$ )

$<2 x$ the upper limit of normal

199

84.7

$>2 x$ the upper limit of normal

36

15.3

Lactic Dehydrogenase (LDH)

Normal range: 110 to 210 units/L)

148

63

$<245$ units/L

87

37

>245 units/L

Procalcitonin

Normal range $(0.15-2 \mathrm{ng} / \mathrm{mL})$

$<2 \mathrm{ng} / \mathrm{mL}$

181

77

$>2 \mathrm{ng} / \mathrm{mL}$

54

23 


\begin{tabular}{|lll|}
\hline Variable & Frequency & Percentage(\%) \\
\hline Neutrophil /Lymphocytic ratio & & \\
$1-3$ & 72 & 30.6 \\
$6-9$ & 89 & 37.8 \\
$>9$ & 74 & 31.4 \\
\hline
\end{tabular}

\subsection{Outcome of the Patients:}

In patients $<65$ years out of 80 patients there were $6(07 \%)$ deaths, $25(31 \%)$ prolong stay and $49(61.25 \%)$ recovered whereas $51(32.9 \%)$ deaths, $82(52.9 \%)$ prolong stay and $22(14.2 \%)$ recovered in time in age group > 65 years. Regarding the gender there were 45 (24.6\%) deaths, 84 (44.91\%) prolong stay, $58(31.0 \%)$ recovery in Male (187) and 12 (25\%) deaths, 23 (45.83\%) prolong stay, 13 (27.0\%) recovery in female (48) group. Severity of illness according to Covid Gram-score in Low- risk category (18) 0 deaths, 03(16.6\%) prolong stay and $15(83.3 \%)$ recovery. Medium risk category (93) 13 (13.9\%) deaths, 26 ( $27.9 \%)$ prolong stay and 54 ( $58 \%$ ) recovery and high risk group (124) $44(35.4 \%)$ deaths, $78(62.9 \%)$ prolog stay and $2(01.6 \%)$ recovery. In no co-morbidity (121) group $7(0.05 \%)$ patients died, $66(54.5 \%)$ has prolong stay and 48 (39.6\%) recovered, in one comorbidity group (49) 14 ( $26.5 \%$ ) deaths, 19 ( $38.7 \%$ ) prolong stay and 16 (32.6\%) recovered, in two comorbidities group (32) 13 (40.6\%) deayhs, 14 ( $43.7 \%$ ) prolong stay and, 5 (15\%) recovery. with three comorbidities group (18) 12(77.7\%) deaths, 4 ( $22.2 \%)$ prolog stay and 2 (11.1\%) recovery and in four comorbidities group (15) 11 (73.3\%) deaths, 04 ( $26.6 \%)$ prolong stay and 0 recovery. Table No: 3 shows the Outcome of patients according to age, gender, severity of illness and co-morbidities.

Table No:3. Outcome of patients according to age,gender,severity of illnessand co-morbidities. 


\begin{tabular}{|llll|}
\hline Variables & Death (57) & Prolong stay (107) & Recovery in Time( 71) \\
\hline Age: & & & \\
$<65(80)$ & $06(07 \%)$ & $25(31.5 \%)$ & $49(61.25 \%)$ \\
\hline $65(155)$ & $51(32.9 \%)$ & $82(52.9 \%)$ & $2214.2 \%)$ \\
\hline Gender & & & \\
Male (187) & $45(24.6 \%)$ & $84(44.91 \%)$ & $58(31.0 \%)$ \\
Female (48) & $12(25 \%)$ & $23(45.83 \%)$ & $13(27.0 \%)$ \\
\hline Severity of Illness: & & & \\
Gram-Covid Score: & & & $15(83.3 \%)$ \\
Low Risk (18) & 0 & $03(16.6 \%)$ & $54(58 \%)$ \\
Moderate Risk (93) & $13(13.9 \%)$ & $26(27.9 \%)$ & $2(01.6 \%)$ \\
High Risk (124) & $44(35.4 \%)$ & $78(62.9 \%)$ & $48(39.6 \%)$ \\
Co-Morbidities: & & & $16(32.6 \%)$ \\
No (121) & $07(0.05 \%)$ & $66(54.5 \%)$ & $05(15 \%)$ \\
One (49) & $14(26.5 \%)$ & $19(38.7 \%)$ & $02(11.1 \%)$ \\
Two (32) & $13(40.6 \%)$ & $14(43.7 \%)$ & 00 \\
Three (18) & $14(77.7 \%)$ & $04(22.2 \%)$ & $04(26.6 \%)$ \\
Four (15) & $11(73.3 \%)$ & & \\
\hline
\end{tabular}

\subsection{Relationship of age, sex, Severity of illness and co-morbidities:}

A strong relationship was observed between the outcome of patient with age, number of co-morbidities, gram - Covid score risk category whereas insignificant relationship with the sex of patients.

AUCROC of age with outcome was $0.65(0.58-0.70)$ with sensitivity of $90 \%$ and specificity of $42 \%$, negative predictive value of $98.7 \%$ and positive predictive value of $64 \%(p=0.010)$.

AUCROC of sex with outcome was $0.51(0.43-0.58)$ with sensitivity of $80 \%$ and specificity of $54 \%$ negative predictive value of $95 \%$ and positive predictive value of $54 \%(p=0.79)$

AUCROC of severity of illness with outcome was $0.69(0.62-0.76)$ with sensitivity of $73 \%$ and specificity of $64 \%$ negative predictive value of $97.9 \%$ and positive predictive value of $96 \%(p=0.010)$.

AUCROC of number of Co-morbidities with outcome was $0.81(0.75-0.88)$ with sensitivity of $88 \%$ and specificity of $64 \%$ negative predictive value of $99 \%$ and positive predictive value of $88 \%(p=0.010)$. 
Table No:4. Shows results of AUCROC (area under curve receiver operating characteristic) showing sensitivity, specificity, positive and negative predictive Between age, Severity of illness, Number of Comorbities, sex with the outcome.Fig. AUCROC(area under curve receiver operating characteristic) showing the relationship between age, Severity of illness, Risk factors, sex with the outcome.

Table No:4. Results of AUCROC (area under curve receiver operating characteristic) showing sensitivity, specificity, positive and negative predictive Between age, Severity of illness, Number of Comorbities, sex with the outcome.

\begin{tabular}{|lllllll|}
\hline Variable & $\begin{array}{l}\text { AUROC } \\
(95 \% \text { Conf:Intervel) }\end{array}$ & $\begin{array}{l}\text { SENSITIVITY } \\
(\%)\end{array}$ & $\begin{array}{l}\text { SPECIFICITY } \\
(\%)\end{array}$ & $\begin{array}{l}\text { NPV } \\
(\%)\end{array}$ & $\begin{array}{l}\text { PPV } \\
(\%)\end{array}$ & P.VALUE \\
\hline AGE & $0.65(0.58-0.70)$ & 90 & 42 & 98.7 & 64 & 0.010 \\
\hline $\begin{array}{l}\text { Severity of } \\
\text { Illness. }\end{array}$ & $0.69(0.62-0.76)$ & 73 & 64 & 97.9 & 96.4 & 0.001 \\
\hline Risk factors & $0.81(0.75-0.88)$ & 88 & 64 & 99.0 & 88.8 & 0.001 \\
\hline SEX & $0.51(0.43-0.58)$ & 22 & 80 & 95.0 & 54 & 0.79 \\
\hline
\end{tabular}

\section{Discussion}

This study included 235 consecutive patients admitted at Liaquat University Hospital from April 2020 to September 2020.

In current study there were $187(79.6 \%)$ male and $48(20.4 \%)$ female. Our results coincide with that of chen et al and wang et.al with a male predominance (73\%) and higher than Jin-jin Zhang with 50,6\% male. ${ }^{7,21,22}$

In a study of 337 patients by Filardo TD et.al comprising 227 male (67.4\%) with a median age 58 years. ${ }^{23}$ Guan W, Ni Z, Hu Y, et in a study of 393 patients 238 (60.6\%) were male and the median age was 62.2 years. ${ }^{24}$

The most prevalent symptoms were fever, breathlessness, cough, anorexia, fatigue in all $235(100 \%)$ patients.

For the first time Huang et.al in a case series of 41 patients noted fever in $98 \%$, cough in $76 \%$ and breathlessness in $55 \%$ cases. ${ }^{25}$ In a study by Garg $S$ et.al comprising 178 patients dyspnea was found in $143(80 \%)$ patients. ${ }^{26} \mathrm{Jain}$ et al. in a meta-analysis of 1813 cases observed cough, fever and fatigue in $70.5 \%, 64.1 \%$ and $66,5 \%$ patients respectively. ${ }^{27}$ Wang $\mathrm{D}$ and colleagues in a study comprising 138 patients observed fever in $136(99 \%)$, fatigue 96 (70\%) and dry cough in 82 (59\%0 patients. ${ }^{21}$

In our study at least one comorbidity was present in 114/235 (48.5\%) .Diabetes mellitus was present in 88/235 (37.4\%), Hypertension in 80/235 (34 \%) patients. Cardiovascular disease (CVD) in 33/235 ( 14\%), 
Chronic Obstructive Airway Disease ( COPD) in 30 /235 ( 12.8), Obesity in 20 / 235 (8.9\%) and renal disease in $14 / 235$ ( $6 \%$ ) patients.

Fei Zhou et al in a study comprising 191 patients observed hypertension 58 (30\%) patients, diabetes mellitus $36(19 \%)$ and coronary heart disease $15(8 \%) .{ }^{28}$

Guan WJ et.al in a study comprising 1590 patients noted hypertension as the most prevalent $(16.9 \%)$ followed by diabetes (8.2\%), cardiovascular disease (3.7\%) and COPD (1.5\%). ${ }^{29}$ Wu et al observed cardiovascular disease in $10.9 \%$, diabetes in $7.3 \%, 6.3 \%$ chronic respiratory disease and $6 \%$ hypertension among 44672 confirmed cases of COVID-19. ${ }^{30}$ In a systemic review and meta-analysis comprising 46248 patients observed hypertension in $17 \pm 7 \%$ patients. ${ }^{31}$ Ruan et.al in a study of 150 covid-19 patients observed hypertension in $43 \%$ patients and cardiovascular disease in $19 \%{ }^{32}$ Guozhen $\mathrm{Li}$, and collegues in a retrospective study of 199 patients revealed diabetes in 76/199 (38.1\%) whereas 123(61.9\%) were non-diabetic. ${ }^{33}$ Age $>65$ years and obesity were noted as two important factors for admission to hospital by Petrilli CM et.al in an analysis of 4103 confirmed cases of CVID - 19. ${ }^{34}$ Zhang $\mathrm{J}$ and collegues in their study included 140 patients found at least one comorbity in $90(68 \%)$ patients. In all those patients the most prevalent co-morbidity was hypertension (30\%) and diabetes mellitus in $12.1 \%$ patients. ${ }^{22}$

In this study severity of illness according to Gram-Covid Score 18 (7.7\%) patients were in low-risk group, 93(39.6\%) in moderate risk group and 124 (52.8\%) in High risk group.

In a cohort by de Terwangne, $C$ et.al patients were classified according to World Health Organization(WHO) severity classification into mild, moderate, severe and critical. No patients in milder disease were admitted. Out of 295 patients 125 (42.4\%) were in moderate,69 (23.4\%) in severe and 101(34.2\%) were critical state. ${ }^{35}$ Liang W et al in a cohort study of 1590 patients divided patients into low ,moderate and high risk based on Gram-Covid score. According to this study 1459 ( $91.7 \%)$ were in non-critical illness and $131\left(8.21 \%\right.$ ) were in critical illness group. ${ }^{17}$ In a study by Wang D and colleagues in a study of 143 patients revealed mild to moderate severity in $72 / 143$ ( $50.3 \%$ ) and severe to critical in $71 / 143(49.6 \%)$ cases. $^{21}$

In our study the outcome showed $57 / 235(24.3 \%$ ) deaths, $107 / 235$ ( $45.5 \%$ ) had prolong stay in hospital and $71 / 235(30.2 \%)$ recovered and discharged from hospital within two weeks time.

In a study of 337 patients by Filardo TD et.al comprising 227 male (67.4\%) with a median age 58 years. The total mortality was $28.9 \%$ inclusive of ICU and Non-ICU patients. ${ }^{36}$ Guan W, Ni Z, Hu Y, et in a study of 393 patients $238(60.6 \%)$ were male and the median age was 62.2 years. Out of total 393 patients 40 (10.2\%) were dead, $260(66.2 \%)$ discharged whereas data for the remaining 93(19.1\%) remain incomplete. ${ }^{29}$ Wang $D$ and colleagues in a study of 143 patients observed that mortality was 14 /143 $(11.2 \%)$ in moderate, $23 / 143(33.3 \%)$ in severe and 68 (67.3\% ) in critical cases. ${ }^{21}$ 
Carfi and colleagues in a study of 143 patients observed that only $13 \%$ patients were symptom free after mean 60 days of disease onset. The most common persistent symptoms were fatigue (53 percent), dyspnea (43 percent), joint pain (27 percent), and chest pain (22 percent); none had fever or features concerning for acute illness. ${ }^{37}$

Tenforde MW and collogues in a multistate telephonic survey of 292 patients having symptoms of cough, fever, breathlessness and fatigue at the time of testing revealed that $65 \%$ patients returned to the baseline health with a median of 7 days( $5-12$ days). Up to $43 \%$ of patients having cough, $35 \%$ with fatigue and $29 \%$ having breathlessness at the time of testing continue to have these symptoms at the $>$ two to three weeks after testing. ${ }^{18}$

A strong relationship was observed between the outcome of patient with age „gram - Covid score risk category and number of comorbidities.

In our study relationship of age with outcome was strong as 6 deaths, 25 prolong stay and 49 recovered were observed in patients $<65$ years of age whereas 51 deaths, 82 prolong stay and 22 recovery in age group $>65$ years. AUCROC of age with outcome was $0.65(0.58-0.70)$ with sensitivity of $90 \%$ and specificity of $42 \%$, negative predictive value of $98.7 \%$ and positive predictive value of $64 \%(p=0.010)$.

In US epidemiologic study a high mortality ranging from 10\%-27\% was observed in the age group 85 and above, $3 \%-11 \%$ in a age group $65-84$ years, $1 \%-3 \%$ in a age group $55-64$ years, and $<1 \%$ in people younger than 55 years. ${ }^{38}$

Harrison S and colleagues in a multicenter study comprising 31461 patients observed the risk of death in patients $>70$ years was 1.3 times greater with heart failure, 1.9 times greater with renal disease compared to those without any these conditions. ${ }^{39}$

In a cohort of 44000 confirmed cases of COVID-19 in China it was revealed that as the age increases the mortality increases with mortality $>80$ years was $14.8 \%, 70-79$ years $8 \%, 60-693.6 \%, 50-591.3 \%, 40-$ 49 years $0.4 \%$ and those younger than 40 years $0.2 \%$. ${ }^{30}$

Regarding the gender there were 45 deaths , 84 prolong stay ,58.recovery in Male and 12 deaths, 23 prolong stay, 13.recovery in female group $(p=0.86)$. AUCROC of sex with outcome was $0.51(0.43-0.58)$ with sensitivity of $80 \%$ and specificity of $54 \%$ negative predictive value of $95 \%$ and positive predictive value of $54 \%(p=0.79)$

In contrast to our study Liu et.al comprising 1190 patients observed death in 157 patients. There were $100 / 157$ (63.7\%) male and 57/157(36.3\%0 female showing high mortality in male comparing to female. ${ }^{40}$ According to a Chinese study the mortality of male were $2.8 \%$ versus $1.7 \%$ for female. ${ }^{41}$ The reasons for high mortality in our study might be due to high number of co-morbidities, more severe illness and more age among female patients. 
In our study the severity of illness according to Covid Gram-score showed in Low- risk category 0 deaths,3 prolong stay and 15 recovery. Medium risk category 13 deaths,26prolong stay and 54 recovery and high risk group 44 deaths, 78 prolog stay and 2 recovery .AUCROC of severity of illness with outcome was $0.69(0.62-0.76)$ with sensitivity of $73 \%$ and specificity of $64 \%$ negative predictive value of $97.9 \%$ and positive predictive value of $96 \%(p=0.010)$.

In a cohort by De Terwangne, $C$ et.al patients were classified according to World Health Organization(WHO) severity classification into mild, moderate, severe and critical. No patients in milder disease were admitted. Out of 295 patients 125 (42.4\%) were in moderate,69 (23.4\%) in severe and $101(34.2 \%)$ were critical state. The mortality was14 (11.2\%) in moderate,23 $(33.3 \%)$ in severe and 68 $(67.3 \%)$ in critical cases. ${ }^{35}$

Liang $W$ et al in a cohort study of 1590 patients divided patients into low ,moderate and high risk based on Gram-Covid score. According to this study 1459 (91.7\%) were in non-critical illness and $131(8.21 \%)$ were in critical illness group. An overall mortality of 50/1590(3.2\%) was observed ${ }^{17}$

In this study the number of comorbidities and outcome showed 7 patients died, 66 has prolong stay and 48 recovered in no co-morbidity, in one comorbidity group 14 deaths, 19 prolong stay and 16 recovered, in two comorbidities group 13 deayhs, 14 prolong stay and, 5 recovery. with three comorbidities group 12 deaths, 4 prolog stay and 2 recovery and in four comorbidities group 11 deaths, 4 prolog stay and 0 recovery. AUCROC of number of Co-morbidities with outcome was $0.81(0.75-0.88)$ with sensitivity of $88 \%$ and specificity of $64 \%$ negative predictive value of $99 \%$ and positive predictive value of $88 \%(p=$ 0.010)

Peng YD et.al in a study of 112 patients dividing them into critical group $(n=16)$ and general group $(n=$ 96) observed higher BMI 25.5 (Cl 23.0, 27.5) in critical group vs general group. 22.0 (Cl 20.0, 24.0), Furthermore on follow up 15/17 (88.4\%) non-survivor had BMI > $25 \mathrm{~kg} / \mathrm{m} 2$ compared to $18 / 95$ (18.24\%) survivors. ${ }^{42}$

In a study by tartof et.al comprising 6916 patients noted a $\mathrm{J}$ shaped association between BMI risk of mortality. According to their observation patients with $\mathrm{BMI}>45 \mathrm{~kg} / \mathrm{m}^{2}$ had 4.18 times and BMI of 40 to 44 $\mathrm{kg} / \mathrm{m}^{2}$ had 2.68 times higher risk of mortality compared to BMl of 18.5 to $24 \mathrm{~kg} / \mathrm{m}^{2}$. This risk was more pronounced in the age group of 60 and below and male gender. ${ }^{43}$

Wang $\mathrm{D}$ in a cohort of 138 hospitalized patients observed any comorbidity in $72 \%$ patients who required intensive care unit (ICU) care (46\% Non-ICU patients) with hypertension in 58\% (31\% in Non- ICU),CVD in $25 \%$ (15\%Non-ICU), Diabetes in $22 \%\left(10 \%\right.$ Non-ICU) patients. ${ }^{22}$

Zhou $\mathrm{F}$ et.al in a cohort study comprising 191 patients comorbidities was revealed in $67 \%$ non-survivors ( $48 \%$ survivors) with hypertension in 48 (30\%in survivors), Diabetes mellitus in $31 \%$ ( $19 \%$ survivors) ,CVD in $13 \%$ ( $8 \%$ survivors) patients. ${ }^{28}$ 
Guan W-j et.al in a cohort of 1099 patients comprising outpatients and inpatients noted any comorbidity in $58 \%$ of non-survivors compared to $24 \%$ in survivor patients with hypertension in $36 \%$ in non-survivors ( $15 \%$ in survivors),Diabetes in $27 \%$ non-survivors ( $7.4 \%$ in survivors) and CVD in $9 \%$ non-survivors (2.5\% survivors). ${ }^{29}$

\section{Conclusion}

COVID-19 patients clinically present with fever, cough and breathlessness. Majority of patients present with moderate to high severity. Patient with advanced age, high risk on gram-Covid score with multiple comorbidities are particularly susceptible to adverse outcome.

\section{Limitation Of Study}

This is a cross sectional study and the results of this study can not be generalized. Prospective cohort studies are needed in local population.

\section{Declarations}

\section{Acknowledgement:}

I am thankful to my Almighty GOD who helped us in finishing us this study. I am thankful to my wife for sacrificing her precocious time allowing me to complete this study. I am also thankful to all fellows working in our group such as Dr.Mumtaz Lakho, Dr.lhsan Rajar, Dr.abdul ghani rahmoon, Prof: Hamid Raza and other fellows of Anesthetic department for their tremendous help. I am thankful to dedicated young doctors particularly Dr.Shahbaz and Dr.Samina for their help.

\section{Authors' contributions:}

This work was carried out in collaboration among all authors. Author S. Shaikh designed the study, performed the statistical analysis, wrote the protocol and wrote the first draft of the manuscript. Authors Fiaza Deedar and Devrajani Tara Chand managed the analyses of the study. Author Devrajani Tara Chand managed the literature searches. All authors read and approved the final manuscript.

\section{Conflict of interest:}

None

\section{Refrences}

1. Zhu N, Zhang D, Wang W, Li X, Yang B, Song J, et al. A novel coronavirus from patients with pneumonia in China, 2019. N Engl J Med. (2020) 382:727-33. doi: 10.1056/NEJMoa2001017 
2. Donnelly CA, Ghani AC, Leung GM, Hedley AJ, Fraser C, Riley S, et al. Epidemiological determinants of spread of causal agent of severe acute respiratory syndrome in Hong Kong. Lancet. (2003) 361:1761-6. doi: 10.1016/S0140-6736(03)13410-1

3. World Health Organization. WHO Director-General's opening remarks at the media briefing on COVID19-11 March 2020. Geneva, Switzerland: World Health Organization; 2020.https://www.who.int/dg/speeches/ detail/who-director-general-s-opening-remarks-at-themedia-briefing- on-covid-19-11-march-2020

4. https://www.who.int/docs/default-source/coronaviruse/situation-reports/20200928-weekly-epiupdate.pdf?sfvrsn=9e354665_65 ( accessed in September 2020)

5. RichardsonS,HirschJS,NarasimhanM,etal;NorthwellCOVID-19 Research Consortium.Presenting characteristics, comorbidities, and outcomes among 5700 patients hospitalized with COVID-19 in the New York City area. JAMA. 2020;323(20):2052-2059. doi:10.1001/jama.2020.6775

6. Rodriguez-MoralesAJ,Cardona-OspinaJA,Gutiérrez, OcampoE ; Latin American NetworkofCoronavirusDisease 2019-COVID-19 Research (LANCOVID-19). Clinical, laboratory and imaging features of COVID-19:a systematic review and meta-analysis. Travel Med Infect Dis. 2020;34:101623. doi:10.1016/j.tmaid.2020.101623.

7. Chen N, Zhou M, Dong X, et al. Epidemiological and clinical characteristics of 99 cases of 2019 novel coronavirus pneumonia in Wuhan, China: a descriptive study. The Lancet 2020. Feb 15;395(10223):507-513. doi: 10.1016/S0140-6736(20)30211-7.

8. Stokes EK, Zambrano LD, Anderson KN, et al. Coronavirus disease 2019 case surveillance-United States, January 22-May 30, 2020. MMWR Morb Mortal Wkly Rep 2020; 69:759-65.

9. Centers for Disease Control and Prevention COVID-19 Response Team. Preliminary estimates of the prevalence of selected underlying health conditions among patients with coronavirus disease 2019United States, February 12- March 28, 2020. MMWR Morb Mortal Wkly Rep 2020; 69:382-6.

10. Shang Y, Pan C, Yang X, Zhong M, Shang $X$, Wu Z, et al. Management of critically ill patients with COVID-19 in ICU: statement from front-line intensive care experts in Wuhan, China. Ann Intensive Care. 2020;10(1):73. https://doi.org/10.1186/s13613-020-00689-1.

11. Force ADT, Ranieri VM, Rubenfeld GD, Thompson BT, Ferguson ND, Caldwell E, Fan E, Camporota L, Slutsky AS. Acute respiratory distress syndrome: the Berlin definition. JAMA. 2012;307(23):252633.https://doi.org/10.1001/jama.2012.5669.

12. American Diabetes Association - Diabetes Care. 2017;40(1):S11 -S24.

13. Unger T, Borghi $C$, Charchar $F$, et al. 2020 international society of hypertension global hypertension practice guidelines[J].J Hypertens 2020, 38(6): 982-1004.

14. Khwaja A. KDIGO clinical practice guidelines for acute kidney injury. Nephron Clin Pract. 2012;120(4):c179-84. https://doi.org/10.1159/000339789.

15. Buist AS, McBurnie MA, Vollmer WM, et al. International variation in the prevalence of COPD (the BOLD Study): a population-based prevalence study. Lancet 2007; 370:741. 
16. Garrow, J.S. \& Webster, J., 1985. Quetelet's index (W/H2) as a measure of fatness. Int. J. Obes., 9(2), pp.147-153.

17. Liang W, Liang H, Ou L, Chen B, Chen A, Li C, Li Y, et.al. China Medical Treatment Expert Group for COVID-19. Development and Validation of a Clinical Risk Score to Predict the Occurrence of Critical Illness in Hospitalized Patients With COVID-19. JAMA Intern Med. 2020 Aug 1;180(8):1081-1089. doi: 10.1001/jamainternmed.2020.2033.

18. Tenforde MW, Billig Rose E, Lindsell CJ. Characteristics of Adult Outpatients and Inpatients with COVID-19 - 11 Academic Medical Centers, United States, March-May 2020. MMWR Morb Mortal Wkly Rep 2020; 69:841.11.

19. Onder G, Rezza G, Brusaferro S. Case-Fatality Rate and Characteristics of Patients Dying in Relation to COVID-19 in Italy. JAMA. 2020 May 12;323(18):1775-1776. doi:

20. Hanley JA, McNeil BJ. A method of comparing the areas under receiver operating characteristic curves derived from the same cases. Radiology. 1983 Sep;148(3):839-43. doi:

10.1148/radiology.148.3.6878708. PMID: 6878708.

21. Wang D, Hu B, Hu C, Zhu F, Liu X, Zhang J, et al. Clinical characteristics of 138 hospitalized patients with 2019 novel coronavirus-infected pneumonia in Wuhan, China. JAMA. (2020) 323:1061-9. doi: 10.1001/jama.2020. 1585

22. Zhang JJ, Dong X, Cao YY, Yuan YD, Yang YB, Yan YQ, Akdis CA, Gao YD. Clinical characteristics of 140 patients infected with SARS-CoV-2 in Wuhan, China. Allergy. 2020 Jul;75(7):1730-1741. doi: 10.1111/all.14238. Epub 2020 Feb 27. PMID: 32077115.

23. Filardo TD, Khan MR, Krawczyk N, Galitzer H, Karmen-Tuohy S, Coffee M, et.al Comorbidity and clinical factors associated with COVID-19 critical illness and mortality at a large public hospital in New York City in the early phase of the pandemic (March-April 2020). PLoS One. 2020 Nov 23;15(11):e0242760. doi:10.1371/journal.pone.0242760. PMID: 33227019; PMCID: PMC7682848.

24. Guan W, Ni Z, Hu Y, et al. Clinical characteristics of coronavirus disease 2019 in China. N Engl J Med 2020;382:1708-1720.

25. Huang C, Wang Y, Li X, et al. Clinical features of patients infected with 2019 novel coronavirus in Wuhan, China. Lancet. 2020; pii: S0140- 6736(20)30183-5. https://doi.org/10.1016/S01406736(20)30183-5. [Epub ahead of print].

26. Garg S, Kim L, Whitaker M, O'Halloran A, Cummings C, Holstein R, et al. Hospitalization Rates and Characteristics of Patients Hospitalized with Laboratory-Confirmed Coronavirus Disease 2019 COVID-NET, 14 States, March 1-30, 2020. MMWR Morb Mortal Wkly Rep. 2020;69(15):458-64.

27. Jain V., Yuan J-M. "Systematic review and meta-analysis of predictive symptoms and comorbidities for severe COVID-19infection." March 16, 2020. Medrxiv: doi.org/10.1101/2020.03.15.20035360.

28. Zhou F, Yu T, Du R, Fan G, Liu Y, Liu Z, Xiang J, et.al. Clinical course and risk factors for mortality of adult inpatients with COVID-19 in Wuhan, China: a retrospective cohort study. Lancet. 2020 Mar 28;395(10229):1054-1062. 
29. Guan WJ, Liang WH, Zhao Y, et al. Comorbidity and its impact on 1590 patients with Covid-19 in China: A Nationwide Analysis. Eur Respir J 2020; in press. doi: 10.1183/13993003.00547-2020.

30. Wu Z, McGoogan JM. Characteristics of and Important Lessons from the Coronavirus Disease 2019 (COVID-19) Outbreak in China: Summary of a Report of 72314 Cases From the Chinese Center for Disease Control and Prevention. JAMA. 2020 Feb 24. doi: 10.1001/jama.2020.2648. [Epub ahead of print]

31. Yang J, Zheng Y, Gou X, Pu K, Chen Z, Guo Q, et al. Prevalence of comorbidities in the novel Wuhan coronavirus (COVID-19) infection: a systematic review and meta-analysis. Int J Infect Dis. 2020 Mar 12. pii: S1201-9712(20)30136-3. doi: 10.1016/j.ijid.2020.03.017. [Epub ahead of print]

32. Ruan Q, Yang K, Wang W, Jiang L, Song J. Clinical predictors of mortality due to COVID-19 based on an analysis of data of 150 patients from Wuhan, China. Intensive Care Med. 2020 Mar 3. doi: 10.1007/s00134-020-05991-x. [Epub ahead of print]

33. Guozhen Li, Qin Deng, Jiali Feng, Fang Li, Nian Xiong, Qiong He, "Clinical Characteristics of Diabetic Patients with COVID-19", Journal of Diabetes Research, vol. 2020, ArticlelD 1652403, 5 pages, 2020.https://doi.org/10.1155/2020/1652403

34. Petrilli CM, Jones SA, Yang J, et al. Factors associated with hospitalization and critical illness among 4,103 patients with COVID-19 disease in New York City. medRxiv. Published online April 11, 2020:2020.04.08.20057794.

35. De Terwangne, C.; Laouni, J.; Jouffe, L.; Lechien, J.R.; Bouillon, V.; Place, S.; Capulzini, L.; Machayekhi, S.; Ceccarelli, A.; Saussez, S.; Sorgente, A.; EPIBASE TEAM, o.b.o. Predictive Accuracy of COVID-19 World Health Organization (WHO) Severity Classification and Comparison with a Bayesian-MethodBased Severity Score (EPI-SCORE). Pathogens 2020, 9, 880.

36. Filardo TD, Khan MR, Krawczyk N, Galitzer H, Karmen-Tuohy S, Coffee M, et.al Comorbidity and clinical factors associated with COVID-19 critical illness and mortality at a large public hospital in New York City in the early phase of the pandemic (March-April 2020). PLoS One. 2020 Nov 23;15(11):e0242760. doi: 10.1371/journal.pone.0242760. PMID: 33227019; PMCID: PMC7682848

37. Carfi A, Bernabei R, Landi F, Gemelli Against COVID-19 Post-Acute Care Study Group. Persistent Symptoms in Patients After Acute COVID-19. JAMA 2020; 324:603.

38. Bialek S, Boundy E, Bowen V, et al. Severe Outcomes Among Patients with Coronavirus Disease 2019 (COVID-19) - United States, February 12-March 16, 2020. MMWR. 2020 Mar 18;69:343-

6. doi:10.15585/mmwr.mm6912e2external icon.

39. Harrison S, Fazio-Eynullayeva E, Lane D, Underhill P, Lip G. Comorbidities associated with mortality in 31,461 adults with COVID-19 in the United States: A federated electronic medical record analysis. PLoS Med. 2020. doi: 10.1371/journal.pmed.1003321.

40. Liu J, Zhang L, Chen Y, Wu Z, Dong X, Teboul JL, Zhang S, Ye X, Liu Y, Wang T, Du H, Li W, Chen D. Association of sex with clinical outcomes in COVID-19 patients: A retrospective analysis of 1190 cases. Respir Med. 2020 Nov;173:106159. doi: 10.1016/j.rmed.2020.106159. Epub 2020 Sep 28. PMID: 33010731; PMCID: PMC7521447. 
41. The Novel Coronavirus Pneumonia Emergency Response Epidemiology Team, The epidemiological characteristics of an outbreak of 2019 novel coronavirus diseases (COVID-19) - China, China CDC Weekly 2 (8) (2020) 113-122. https://doi: 10. 46234/ccdcw2020.032.

42. Peng YD, Meng K, Guan HQ, et al. [Clinical characteristics and outcomes of 112 cardiovascular disease patients infected by 2019-nCoV]. Zhonghua Xin Xue Guan Bing Za Zhi. 2020;48(0):E004. [PMID:32120458

43. Tartof SY, Qian L, Hong V, et al. Obesity and Mortality Among Patients Diagnosed With COVID-19: Results From an Integrated Health Care Organization. Ann Intern Med. 2020;173(10):773-781. doi:10.7326/M20-3742

\section{Figures}




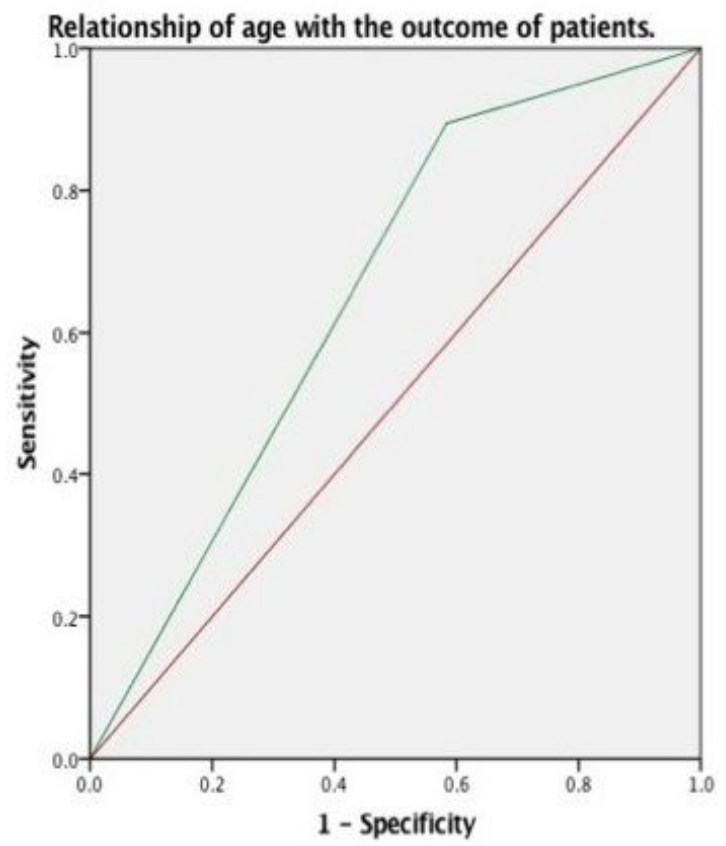

Diagonal segments are produced by ties.

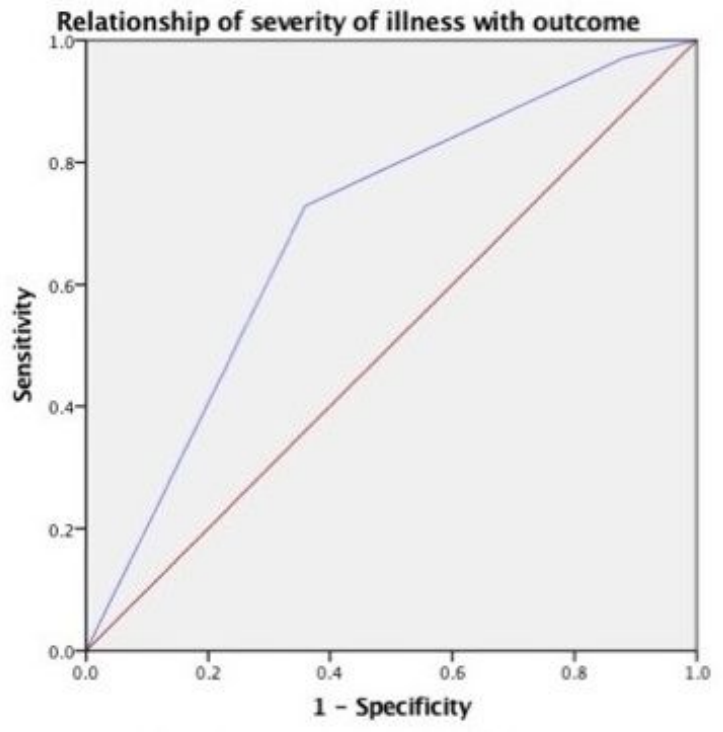

Diagonal segments are produced by ties.

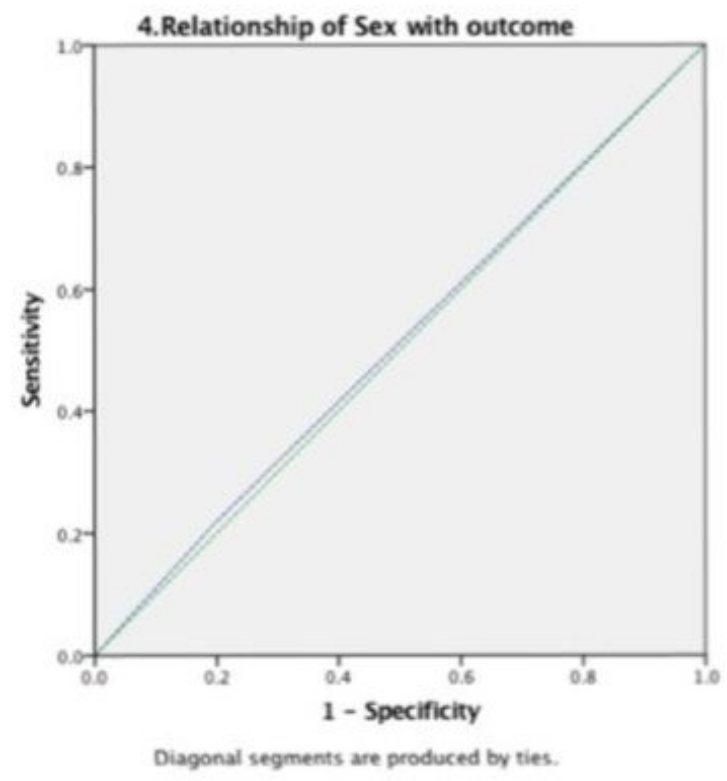

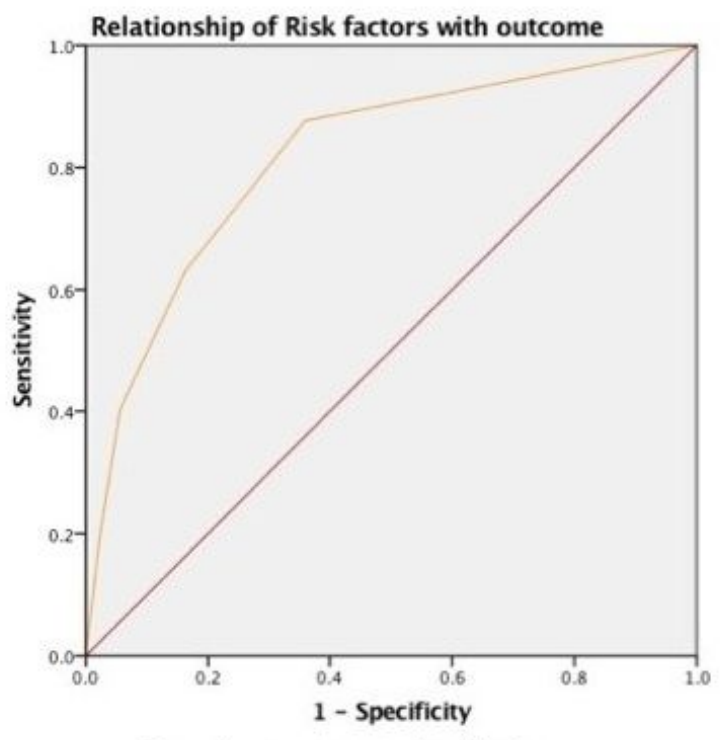

Diagonal segments are produced by ties.

\section{Figure 1}

AUCROC (area under curve receiver operating characteristic) showing the relationship between age, Severity of illness, Risk factors, sex with the outcome. 